\title{
Proton-helium elastic scattering: a possible high-energy polarimeter at RHIC-BNL
}

\author{
C. Bourrely and J. Soffer \\ Centre de Physique Théorique - CNRS - Luminy, \\ Case 907 F-13288 Marseille Cedex 9 - France
}

\begin{abstract}
We examine a suggestion to use $\mathrm{p}^{4} \mathrm{He}$ elastic scattering, as an absolute polarimeter for high-energy polarized proton beams, by means of a CoulombNuclear Interference effect for the single-spin asymmetry $A_{N}(t)$, around the diffractive minimum of the differential cross section $|t| \sim 0.21 \mathrm{GeV}^{2}$. Although this reaction has a fairly simple dynamical structure, our theoretical uncertainties and the present experimental inaccuracy of the differential cross section in this $t$ region, allows one to generate dramatic effects for $A_{N}(t)$, which will be discussed.
\end{abstract}

PACS numbers: 13.85.Dz, 12.90.+b, 13.88.+e

Key-Words : Proton-Helium elastic scattering, spin asymmetry, polarimeter.

Number of figures : 4

September 1998

Unité Propre de Recherche 7061

CPT-98/P.3688

Web address: www.cpt.univ-mrs.fr 
High-energy polarized proton beams are under construction at $R H I C-B N L$ and thanks to the so-called Siberian snake technique, the beam polarization is expected to reach the value $P=70 \%$ and to be maintained at this impressive high level. This is one of the key elements supporting the vast spin phenomena programme for $p p$ collisions, which will be undertaken in the near future [1]. It has also motivated some detailed studies at DESY in order to decide whether or not HERA could operate as a ep collider with both electron and proton beams polarized [2]. However one should have a reliable method for measuring $P$ and the primary goal would be to achieve an accuracy of $5 \%$ or better, i.e. $\Delta P / P \leq 0.05$. This important calibration problem has given rise recently to some activity, several methods have been proposed and their limitations have been discussed [3]. In particular, one interesting candidate is the so-called Coulomb-Nuclear Interference (CNI) polarimeter relying on an idea, first suggested by Schwinger [4. If we consider $p p$ elastic scattering near the forward direction, say for $|t| \sim 10^{-3} \mathrm{GeV}^{2}$, the single-spin asymmetry $A_{N}(t)$ arises primarily from the interference between the real electromagnetic helicity-flip amplitude and the imaginary hadronic helicity-nonflip amplitude. It can be calculated exactly [5, 6] and one finds that it has a maximum value of about $4 \%$ for $|t| \sim 3.10^{-3} \mathrm{GeV}^{2}$, which is almost energy independent. This effect has been investigated by the E-704 experiment at FNAL at $p_{l a b}=200 \mathrm{GeV} / \mathrm{c}$ [7] and the results are consistent with the theoretical prediction. However the situation is not as simple as that, because the hadronic interaction need not conserve helicity in the small $t$ region and the existence of a non-zero single-flip hadronic amplitude introduces a substantial uncertainty on the predicted asymmetry [8, 9, 10]. Unfortunately, the lack of accuracy in the E-704 experiment leaves too much freedom on the size of the singleflip hadronic amplitude and as a result, given the present data, the CNI polarimeter is not a method which can achieve the desired $5 \%$ beam polarization error goal.

Another polarimetry method which involves $\mathrm{p}-{ }^{4} \mathrm{He}$ elastic scattering has been first briefly suggested in Ref. [3] and we think it deserves a careful phenomenological analysis, which is presented in this paper. Since ${ }^{4} \mathrm{He}$ is a spinless object, p- ${ }^{4} \mathrm{He}$ elastic scattering is a simple reaction which is described in terms of two helicity amplitudes, the nonflip $\phi_{+}(t)$ and the flip $\phi_{-}(t)$. The differential cross section reads

$$
\frac{d \sigma(t)}{d t}=\left|\phi_{+}(t)\right|^{2}+\left|\phi_{-}(t)\right|^{2}
$$

and the single-spin asymmetry is

$$
A_{N}(t)=\frac{2 \operatorname{Im}\left[\phi_{+}(t) \phi_{-}(t)^{*}\right]}{\left|\phi_{+}(t)\right|^{2}+\left|\phi_{-}(t)\right|^{2}} .
$$

$\phi_{+}$and $\phi_{-}$are written in terms of hadronic and electromagnetic amplitudes in the form $\phi_{ \pm}(t)=\phi_{ \pm}^{h}(t)+e^{i \delta} \phi_{ \pm}^{e}(t)$, where $\delta$ is the Coulomb phase shift. We have

$$
\phi_{+}^{e}(t)=-\frac{4 \alpha \sqrt{\pi}}{|t|} G_{p}(t) G_{H e}(t)
$$

where $\alpha$ is the fine-structure constant, $G_{p}(t)$ is the proton electromagnetic form factor $G_{p}(t)=1 /(1+|t| / 0.71)^{2}$ and $G_{H e}(t)=\left[1-(2.56 t)^{6}\right] e^{11.70 t}$ is the ${ }^{4}$ He electromagnetic form factor [11]. Similarly we have

$$
\phi_{-}^{e}(t)=\sqrt{|t|} \frac{\mu_{p}-1}{2 m_{p}} \phi_{+}^{e}(t),
$$


where $\mu_{p}$ is the magnetic moment of the proton and $m_{p}$ its mass.

Our theoretical knowledge of $\phi_{ \pm}^{h}(t)$ is less straightforward, but before going into this discussion, let us briefly review the experimental situation. At low energies, say, $1.1 \leq p_{l a b} \leq 2.5 \mathrm{GeV} / \mathrm{c}$, the differential cross section and the single-spin asymmetry have been accurately measured at the $Z G S$-Argonne [12], using polarized proton beams. The cross section has a diffractive minimum around $|t|=0.21 \mathrm{GeV}^{2}$ and $A_{N}(t)$, which is large (40-50\%), exhibits also an interesting behavior in the dip region. At higher energies, say, $45 \leq p_{l a b} \leq 400 \mathrm{GeV} / \mathrm{c}$, only $d \sigma / d t$ has been measured [13], and the diffractive minimum remains essentially at the same $t$ value (see Fig. (1).

Since we are concerned by the proton beams at $R H I C-B N L$, whose momentum lie between $50 \mathrm{GeV} / \mathrm{c}$ and $250 \mathrm{GeV} / \mathrm{c}$, we will concentrate on the high-energy data. Several analysis of these data have been made in the past based on, for example, a Chou-Yang type model [14] or a Glauber model [13], but here we will present a rather simple phenomenological model. A Regge exchange approach is greatly simplified by the fact that, since ${ }^{4} \mathrm{He}$ is an isoscalar, the isovector " $\rho$ exchange", which has a large flip coupling, is forbidden. Moreover only isoscalar trajectories can be exchanged. They contribute mainly to the non-flip amplitude $\phi_{+}(t)$ and the Pomeron prevails at very high-energy. Consequently, one can assume the dominance of a purely diffractive Pomeron of the form $A e^{B t}$, at fixed high-energy 1 . Therefore, as a first approximation, we take the simple parametrization

$$
\operatorname{Im} \phi_{+}^{h}(t)=A e^{B t}-C e^{D t},
$$

where the second term stands for rescattering effects, so we expect $C \ll A$ and $D \ll B$. For the moment we neglect $\operatorname{Re} \phi_{+}^{h}(t)$ and $\phi_{-}^{h}(t)$, but we will come back to them later. The fit of the cross section data at $E_{l a b}=393 \mathrm{GeV}$, shown in Figs. 1-2, is excellent and leads to the following values of the parameters

$$
A=31.84 \sqrt{m b} / G e V, B=15.51 G e V^{-2}, C=3.69 \sqrt{m b} / G e V, D=5.68 G e V^{-2} .
$$

We have also well fitted the total cross section $\mathrm{p}-{ }^{4} \mathrm{He}$ value, namely $\sigma_{\text {tot }}=(125.9 \pm$ $0.6) m b$. Note that in this case, $\operatorname{Im} \phi_{+}^{h}(t)$ changes sign at $|t|=0.219 G e V^{2}$, so we find a very deep diffractive minimum, namely $d \sigma / d t=5.10^{-5} \mathrm{mb} / \mathrm{GeV}^{2}$ at this $|t|$ value, which is due to the contributions of $\phi_{ \pm}^{e}(t)$. Although the data are not very accurate in this region, it would be surprizing to get such a small cross section, but one cannot rule out such a possibility 7 . If we now calculate $A_{N}(t)$, it is driven by the product $\operatorname{Im} \phi_{+}^{h}(t) \cdot \phi_{-}^{e}(t)$ and the result is depicted by the solid line in Fig. 3. In the very small $|t|$ region we check that we have the usual CNI effect at the level of $4 \%$ or so, and in the vicinity of the dip, we find a strong oscillation between $+35 \%$ and $-35 \%$, which is better displayed in Fig. 1 .

As already mentioned above, there is no fundamental theoretical reason to believe that $\phi_{-}^{h}(t)=0$, even in a dynamical framework where the Pomeron dominates. This

\footnotetext{
${ }^{1}$ The energy dependence and the phase of the Pomeron, which have been obtained in a very successful analysis of $p p$ and $\bar{p} p$ elastic scattering [15, could be also used here, but it goes beyond the scope of this paper.

${ }^{2}$ Assuming a conservative ${ }^{4} \mathrm{He}$ jet density and a realistic proton beam intensity, the luminosity is expected to be high enough, to allow such a measurement with a reasonable accuracy (W.Guryn, private communication).
} 
important issue of the size of the Pomeron flip coupling has been studied in details [16, 17] and if we take, in analogy with Eq. (4),

$$
\operatorname{Im} \phi_{-}^{h}(t)=r \frac{\sqrt{|t|}}{m_{p}} \operatorname{Im} \phi_{+}^{h}(t),
$$

one finds from different arguments a value of $r$ of $10 \%$ or below. We have also included such a contribution in our fit of $d \sigma / d t$ and the best fit leads to $r=0.25$, with almost no changes in A, B, C and D. This new contribution fills up the dip in the cross section, as show in Fig. 2 (dotted line) and we see in Figs. 3 -1 that the strong oscillation of $A_{N}(t)$ is now replaced by a smooth curve with a maximum value of $13 \%$ or so, in the dip region.

This situation is somehow oversimplified because, so far, we have neglected the fact that from the data [13], one can extract $\rho$, the ratio of the real to the imaginary part of the forward scattering amplitude and they find $\rho=+0.102 \pm 0.035$. Therefore it is clear that we should take $\operatorname{Re} \phi_{+}^{h}(0) \neq 0$. We don't know the $t$-dependence of $R e \phi_{+}^{h}(t)$ but for simplicity we will assume it has the slope B of the leading term of $\operatorname{Im} \phi_{+}^{h}(t)$. So if we now take

$$
\operatorname{Re} \phi_{+}^{h}(t)=E e^{B t}
$$

the best fit leads to $E=3.09 \sqrt{m b} / G e V$. The net effect of this real part is also to fill up the dip, as show in Fig. 2. We have first considered the case where $r=0$ (small dashed curve) and a second case with $r \neq 0$, which was fitted and led to $r=0.15$ (dotteddashed curve). This value is much smaller than that found above, in the absence of $R e \phi_{+}^{h}(t)$. These two cases correspond to very different predictions for $A_{N}(t)$ as shown in Figs. 3. 3 . In the first case $A_{N}(t)$ is a smooth curve (small dashed line) which changes sign at the dip position and in the second case it is very large and reaches almost $-100 \%$. This effect is entirely due to the product of $\operatorname{Re} \phi_{+}^{h}(t)$ and $\operatorname{Im} \phi_{-}^{h}(t)$, which dominate and become almost equal in magnitude at the dip position. Since the sign of $\operatorname{Im} \phi_{-}^{h}(t)$ is unknown, by changing this sign one can have the mirror effect. In this case, it is no longer a CNI effect.

Finally, one can envisage another realistic situation, where $R e \phi_{+}^{h}(t)$ has not the same t-dependence as $\operatorname{Im} \phi_{+}^{h}(t)$. This is the case in various models and in particular in Ref. [15], where the real part decreases faster than the imaginary part. So we have used again eq.(8) with a larger value of the slope, that is $B=20 \mathrm{GeV}^{-2}$, and $r=0.15$. The results are shown in Figs. 2-3-4, by the large dashed lines. As expected, the filling of the dip is less pronounced than with the previous value $B=15.51 \mathrm{GeV}^{-2}$. The shape of $A_{N}(t)$ is not affected, but its magnitude is reduced accordingly.

To summarize, this phenomenological study of $\mathrm{p}-{ }^{4} \mathrm{He}$ elastic scattering, shows that this simple reaction cannot be easily used as an absolute polarimeter. An accurate measurement of $d \sigma / d t$ in the dip region, might help us to pin down the value of $R e \phi_{+}^{h}(t)$, but in order to clearly disentangle its effect, on the filling of the dip, from that of $\operatorname{Im} \phi_{-}^{h}(t)$, one certainly needs a direct measurement of $A_{N}(t)$, which hopefully, will have large values in the dip region.

We are grateful to Boris Kopeliovich and Tai Tsun Wu for several helpful discussions. 


\section{References}

[1] Proceedings of the Workshop RHIC Spin Physics, Riken BNL Research Center, April 27-29 (1998) (vol.7, Ed. T.D. Lee) report BNL-65615 and references therein.

[2] Proceedings of the Workshop Deep Inelastic Scattering off Polarized Targets:Theory meets Experiment, DESY-Zeuthen, September 1-5 (1997) (Eds. J. Blümlein, A. De Roeck, T. Gehrmann and W.-D. Nowak) report DESY 97-200 and references therein.

[3] B.Z. Kopeliovich, High-Energy Polarimetry at RHIC, hep-ph/9801414.

[4] J. Schwinger, Phys. Rev. 73, 407 (1948).

[5] B.Z. Kopeliovich and I.I. Lapidus, Sov.J.Nucl. Phys. 19, 114 (1974).

[6] N.H. Buttimore, E. Gotsman and E. Leader, Phys. Rev. D18, 694 (1978).

[7] N. Akchurin et al., Phys. Lett. 229B, 299 (1989); Phys. Rev. D48, 3026 (1993).

[8] B.Z. Kopeliovich and B.G. Zakharov, Phys. Lett. 226, 156 (1989).

[9] L.T. Trueman, preprint BNL-63700, hep-ph/9610429.

[10] C. Bourrely and J. Soffer, Proceedings of the "12th Int. Symp. on High-energy Spin Physics", Amsterdam Sept.10-14 (1996), World Scientific (1997) p.825 (Eds. C.W. de Jager et al.).

[11] J.S. Mc Carthy et al., Phys. Rev. C15, 1396 (1977).

[12] R. Klem et al., Phys. Rev. Lett. 22,1272 (1977); Phys. Lett. 70B, 155 (1977).

[13] A. Bujak et al., Phys. Rev. D23, 1895 (1981).

[14] R.J. Lombard and A. Tellez-Arenas, Phys. Lett. 165B, 205 (1985).

[15] C. Bourrely, J. Soffer and T.T. Wu, Proceedings of the VIth Blois Workshop, Blois, 20-24 June 1995, Editions Frontières 1996, p.15 and references therein.

[16] Proceedings of the Workshop Hadron Spin-Flip at RHIC Energies, Riken BNL Research Center, July 21-August 22 (1997) (vol.3, Ed. T.D. Lee) report BNL64724 and references therein.

[17] N.H. Buttimore, B.Z. Kopeliovich, E. Leader, J. Soffer and L.T. Trueman, preprint CPT-98/P.3693 (in preparation). 


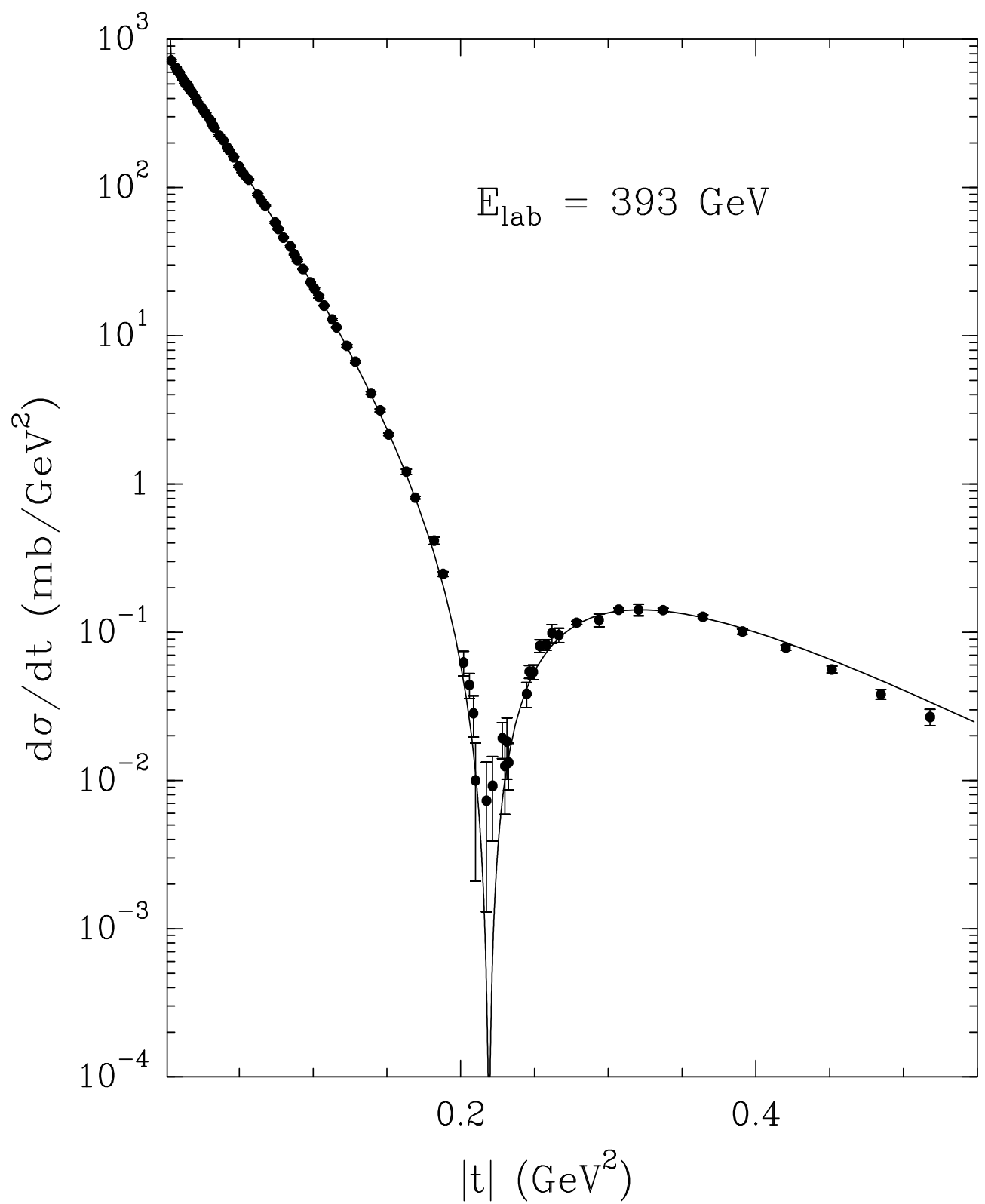

Figure 1: Differential cross section for $\mathrm{p}^{-}{ }^{4} \mathrm{He}$ at $E_{l a b}=393 \mathrm{GeV}$ as a function of $|t|$. Data are from Ref. [13]. The solid line is the result of our fit using eqs.(5) and (66). 


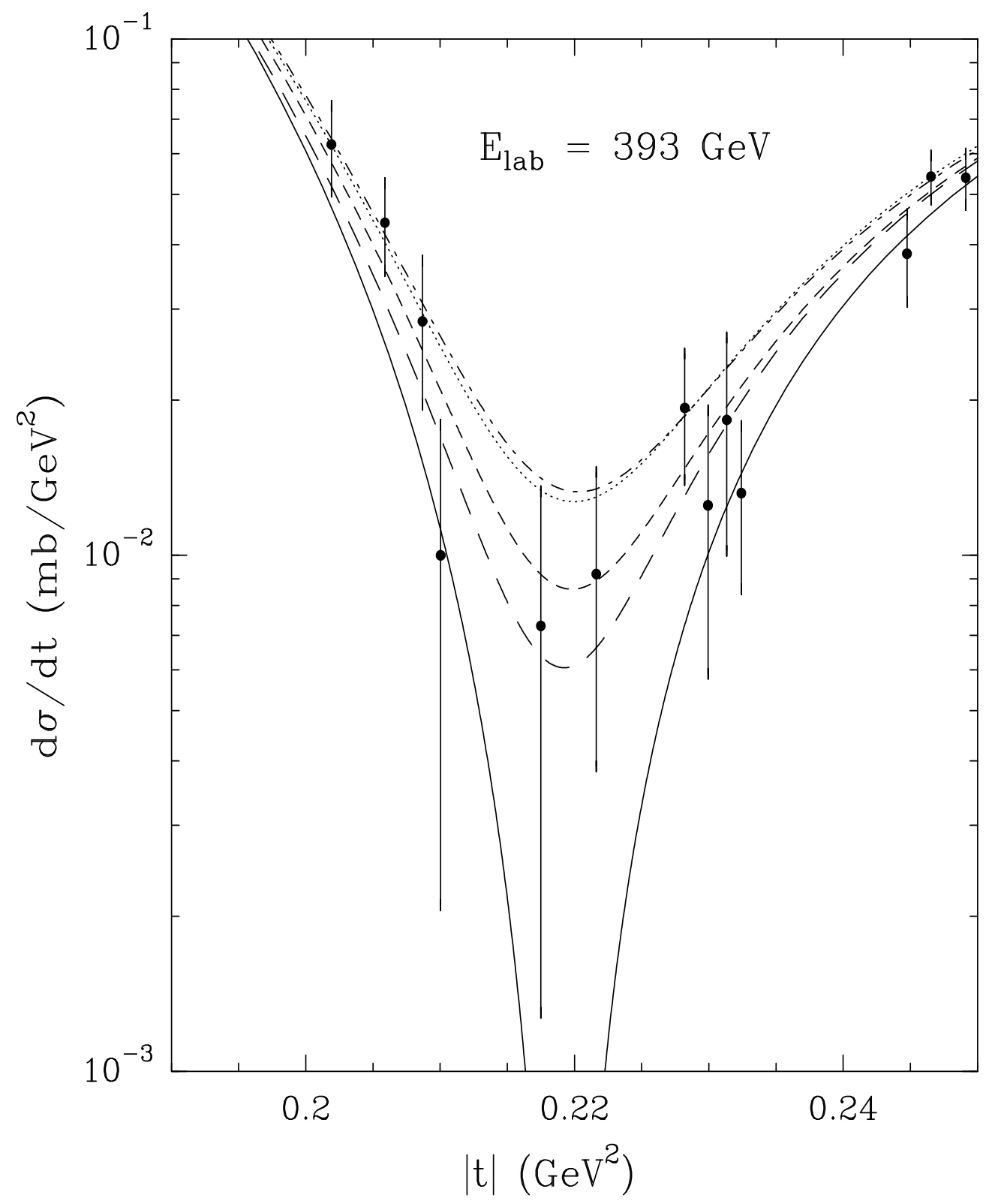

Figure 2: Enlarged dip region of Fig. 1, showing different possibilities described in the text. 


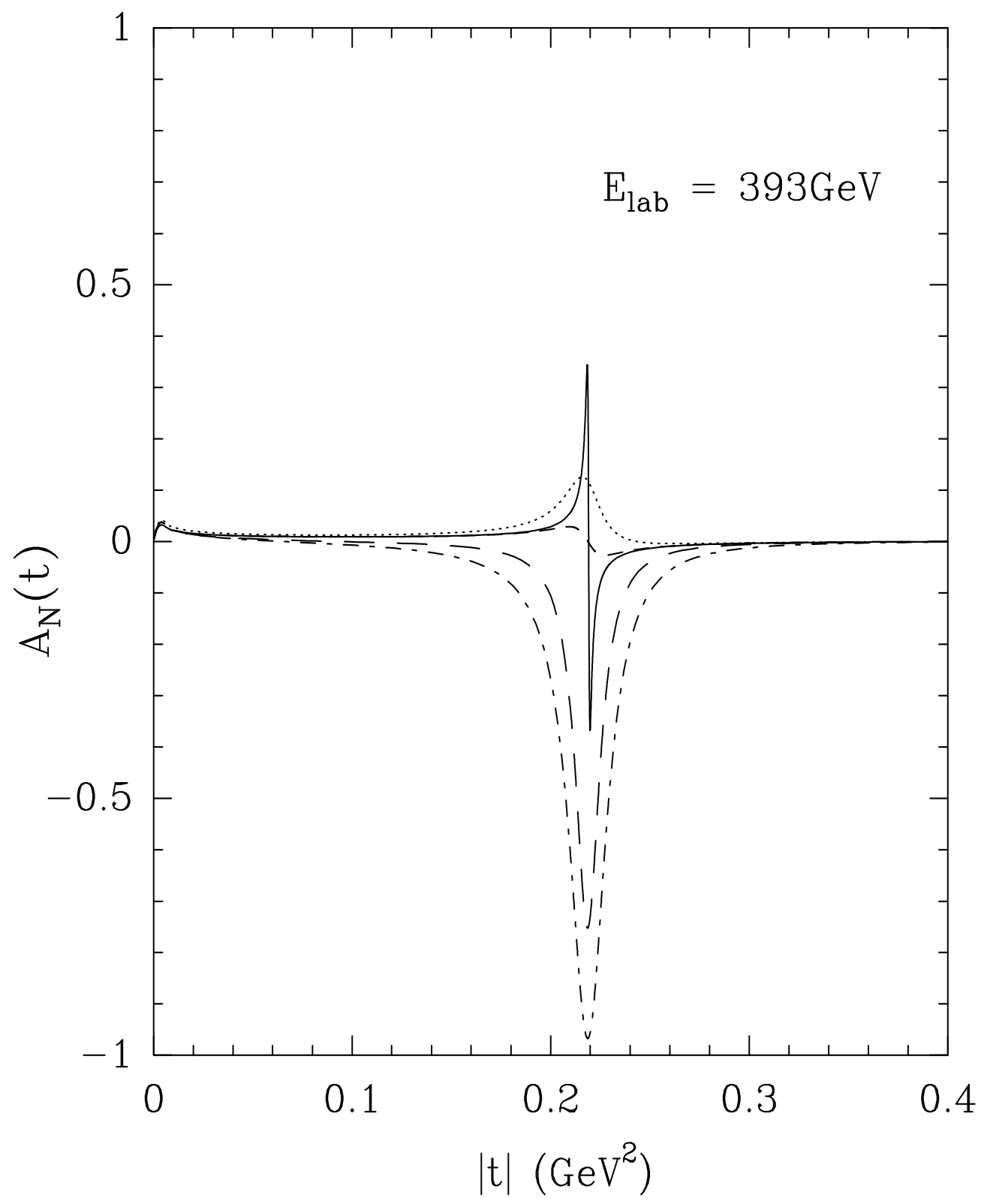

Figure 3: Single-spin asymmetry $A_{N}(t)$ for $\mathrm{p}^{4} \mathrm{He}$ at $E_{l a b}=393 \mathrm{GeV}$ as a function of $|t|$, showing different predictions explained in the text. 


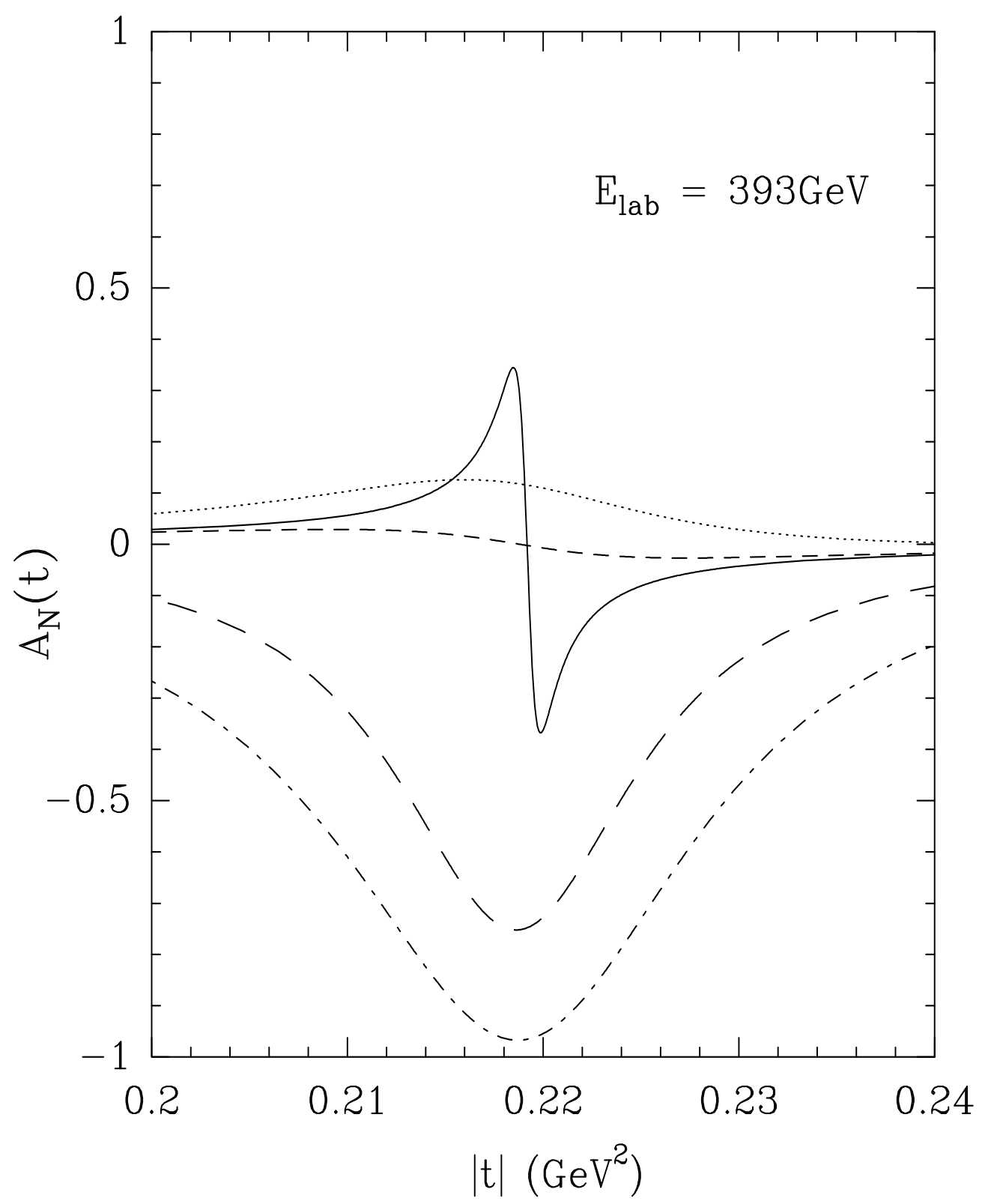

Figure 4: Enlarged dip region of Fig. 3. 